\title{
A NULLSET FOR NORMAL FUNCTIONS IN SEVERAL VARIABLES
}

\author{
JUHANI RIIHENTAUS
}

(Communicated by Clifford J. Earle, Jr.)

\begin{abstract}
Suppose that $\Omega$ is a domain in $C^{n}, E \subset \Omega$ is closed in $\Omega$, and $f: \Omega \backslash E \rightarrow \mathbf{C}^{*}$ is a meromorphic function. We show that if $f$ is normal and $E$ is an analytic subvariety or, more generally, of locally finite $(2 n-2)$ dimensional Hausdorff measure in $\Omega$ satisfying a certain geometric condition, then $f$ can be extended to a meromorphic function (= holomorphic mapping) $f^{*}: \Omega \rightarrow \mathbf{C}^{*}$. In the case of a subvariety sufficient, but not necessary, for the geometric condition is that the singularities of $E$ are normal crossings. As a digression, we give a new proof for the following result, due to Parreau in the case $n=1$ : if $f$ is in the Nevanlinna class and $E$ is polar (in $R^{2 n}$ ), then $f$ has a meromorphic extension $f^{*}$ to $\Omega$.
\end{abstract}

\section{INTRODUCTION}

The following is a result of Lehto and Virtanen:

1.1. Theorem [LV, Theorem 9, p. 62]. Suppose that $G$ is a domain in $\mathbf{C}$ and $a \in G$. If $f: G \backslash\{a\} \rightarrow \mathbf{C}^{*}$ is a normal meromorphic function, then $f$ has a meromorphic extension $f^{*}: G \rightarrow \mathbf{C}^{*}$.

For a slightly different proof see [Jä, Lemma 1, p. 1172]. Using the above result and induction, Järvi [Jä, Theorem 2, p. 1174] generalized Lehto's and Virtanen's result to several variables by showing that an analytic subvariety is removable for normal meromorphic functions. Järvi further showed [Jä, Theorem 1, p. 1173] that if the exceptional analytic subvariety is of codimension 1 and its singularities are normal crossings, then the extension is in fact a meromorphic function to $\mathbf{C}^{*}$; i.e., the indeterminancy set of the extended function is empty. (Järvi uses in this connection the term "holomorphic mapping to $\mathbf{C}^{*}$.")

In Theorems 3.5 and 3.9 below we generalize Järvi's results by pointing out that it is sufficient that the exceptional set is of locally finite $(2 n-2)$-dimensional Hausdorff measure and moreover, in the generalization of Järvi's Theorem 1, satisfies a certain geometric condition. In the proof we need (implicitly) another removable singularity result, Theorem 3.1 below. As another application of

Received by the editors February 21, 1989 and, in revised form, August 18, 1989.

1980 Mathematics Subject Classification (1985 Revision). Primary 32H25, 32D20; Secondary 30D45, 30D50.

Key words and phrases. Normal function, Nevanlinna class. 
this theorem, we obtain in Corollary 3.2 below a new, unified proof for the Nevanlinna class results [Pa, Théorème 20, p. 182] and [HR1, Theorem 3.4, p. 477]. For a previous result see [CG1, Theorem C, p. 241].

1.2. We use Cima's and Krantz's definition for normal meromorphic functions [CK, pp. 305-306], see also [Jä, p. 1171]. Note that other definitions exist also. We use mainly the same notation as in [HR1], [HR2] and [Ri2]. Nevertheless, for the sake of convenience of the reader, we recall the following.

The spherical metric in the extended complex plane $\mathbf{C}^{*}$ is denoted by $q$. We identify $C^{n}\left(=\mathbf{C}^{n}\right)$ with $R^{2 n}\left(=\mathbf{R}^{2 n}\right), n \geq 1$. We write $B^{2 n}\left(z_{0}, r\right)$ for the open ball in $C^{n}$ with center $z_{0}$ and radius $r$. If $z=\left(z_{1}, \ldots, z_{n}\right) \in C^{n}$, $n \geq 2, A \subset C^{n}$, and $1 \leq j \leq n$, then we write

$$
Z_{j}=\left(z_{1}, \ldots, z_{j-1}, z_{j+1}, \ldots, z_{n}\right), \quad z=\left(z_{j}, Z_{j}\right)
$$

and

$$
\begin{gathered}
A\left(Z_{j}\right)=\left\{z_{j} \in \mathbf{C}: z=\left(z_{j}, Z_{j}\right) \in A\right\}, \\
A\left(z_{j}\right)=\left\{Z_{j} \in C^{n-1}: z=\left(z_{j}, Z_{j}\right) \in A\right\}
\end{gathered}
$$

for the sections of $A$. The $\alpha$-dimensional Hausdorff outer measure is denoted by $H^{\alpha}$. Recall that $H^{0}(A)=\operatorname{card} A$ is the number of points of $A$. Suppose that $\Omega$ is a domain in $C^{n}, n \geq 1$. It is well-known that if $V$ is a $k$-dimensional analytic subvariety in $\Omega$, then $H^{2 k}(V \cap K)<\infty$ for each compact set $K \subset \Omega$. Suppose that $f \not \equiv \infty$ is meromorphic in $\Omega$. Then there are analytic subvarieties $P_{f}$, the pole set of $f$, and $I_{f}$, the indeterminancy set of $f$, in $\Omega$ such that $I_{f} \subset P_{f}, H^{2 n-4}\left(I_{f} \cap K\right)<\infty, H^{2 n-2}\left(P_{f} \cap K\right)<\infty$ for each compact set $K \subset \Omega, f$ is holomorphic in $\Omega \backslash P_{f}, f$ is spherically continuous in $\Omega \backslash I_{f}$, and $f \mid P_{f} \backslash I_{f}=\infty$. In the sequel we use the following convention: When we write "...f: $\Omega \rightarrow \mathbf{C}^{*}$ is a meromorphic function ..." or "... a meromorphic function with values in $\mathbf{C}^{*} \ldots$ " or something equivalent, then it is always meant that $f$ is meromorphic in $\Omega$ and $I_{f}=\varnothing$, i.e., locally in $\Omega$ either $f$ or $1 / f$ is holomorphic. Our terminology (probably nonstandard) here follows that used by Cima and Krantz [CK, p. 305]. As noted above, the term "holomorphic mapping to $\mathbf{C}^{* *}$ is also used; see, e.g., [Jä and $\mathrm{Ki}$ ].

\section{LEMMAS}

In this section we give the lemmas we need in the sequel.

2.1. Lemma (cf. [Fe, 2.10.25, 2.10.11, pp. 188, 176] and [Sh, Corollary 4, p. 114]). Suppose that $E \subset C^{n}, n \geq 2$, and $1 \leq j \leq n$.

(a) If $H^{2 n-1}(E)=0$, then $H^{1}\left(E\left(Z_{j}\right)\right)=0$ for $H^{2 n-2}$-almost all $Z_{j} \in$ $C^{n-1}$.

(b) If $H^{2 n-2}(E)<\infty$, then $H^{2 n-4}\left(E\left(z_{j}\right)\right)<\infty$ for $H^{2}$-almost all $z_{j} \in \mathbf{C}$ and $H^{0}\left(E\left(Z_{j}\right)\right)<\infty$ for $H^{2 n-2}$-almost all $Z_{j} \in C^{n-1}$. 
The following is a special case of Cima's and Krantz's result [CK, Corollary 1.7, p. 309]; the statement concerning the order of normality (see [CK, definition for normality, pp. 305-306]) is implicit in this cited result. See also [Jä, Lemma 2, p. 1173].

2.2. Lemma. Suppose that $\Omega$ is a domain in $C^{n}, n \geq 2$, and $1 \leq j \leq n$. Suppose that $f: \Omega \rightarrow \mathbf{C}^{*}$ is a normal meromorphic function. If $Z_{j} \in C^{n-1}$ is such that $\Omega\left(Z_{j}\right)$ is a nonempty domain in $\mathbf{C}$, then the meromorphic function $f_{Z_{j}}: \Omega\left(Z_{j}\right) \rightarrow \mathbf{C}^{*}$,

$$
f_{Z_{j}}\left(z_{j}\right)=f\left(z_{j}, Z_{j}\right),
$$

is normal. Similarly, if $z_{j} \in \mathbf{C}$ is such that $\Omega\left(z_{j}\right)$ is a nonempty domain in $C^{n-1}$ and $f_{z_{j}}: \Omega\left(z_{j}\right) \rightarrow \mathbf{C}^{*}$,

$$
f_{z_{j}}\left(Z_{j}\right)=f\left(z_{j}, Z_{j}\right),
$$

is a meromorphic function, then $f_{z_{j}}$ is normal. Moreover, $C_{f_{z_{j}}}, C_{f_{z_{j}}} \leq C_{f}$, where $C_{f_{z_{j}}}, C_{f_{z_{j}}}$, and $C_{f}$ are the orders of normality of $f_{Z_{j}}, f_{z_{j}}$, and $f$, respectively.

For the next lemma see, e.g., [HR2, Lemma 3.4, p. 299].

2.3. Lemma. Suppose that $\Omega$ is a domain in $C^{n}, n \geq 2, E \subset \Omega$ is closed in $\Omega$ and $f$ is holomorphic in $\Omega \backslash E$. If for each $j, 1 \leq j \leq n$, and for $H^{2 n-2}$-almost all $Z_{j} \in C^{n-1}$, the section $E\left(Z_{j}\right)$ is totally disconnected and the holomorphic function $f_{Z_{j}}:(\Omega \backslash E)\left(Z_{j}\right) \rightarrow \mathbf{C}$,

$$
f_{Z_{j}}\left(z_{j}\right)=f\left(z_{j}, Z_{j}\right) \text {, }
$$

has a meromorphic extension to $\Omega\left(Z_{j}\right)$, then $f$ has a meromorphic extension to $\Omega$.

2.4. Lemma [Ca, p. 202]. Let $\mathscr{F}$ be a family of functions meromorphic in a domain $G$ of $\mathbf{C}$. Suppose that each $f \in \mathscr{F}$ omits three distinct points $a_{f}, b_{f}, c_{f} \in \mathbf{C}^{*}$. If there is a $\delta>0$ such that

$$
q\left(a_{f}, b_{f}\right) q\left(b_{f}, c_{f}\right) q\left(c_{f}, a_{f}\right) \geq \delta
$$

for all $f \in \mathscr{F}$, then $\mathscr{F}$ is spherically equicontinuous.

\section{Singularities}

We begin with a partial generalization to [HR2, Theorem 3.5, pp. 300-301]. For a related result see [Ri2, Theorem 2, p. 549].

3.1. Theorem. Suppose that $\Omega$ is a domain in $C^{n}, n \geq 1$, that $E \subset \Omega$ is closed in $\Omega$ and $H^{2 n-1}(E)=0$. Suppose that $f$ is holomorphic in $\Omega \backslash E$. If 
for some $p \in \mathbf{R}$

$$
\int_{\Omega \backslash E} \frac{|f(z)|^{p-2}}{\left(1+|f(z)|^{p}\right)^{2}} \sum_{j=1}^{n}\left|\frac{\partial f}{\partial z_{j}}(z)\right|^{2} d m(z)<\infty,
$$

then $f$ has a meromorphic extension $f^{*}$ to $\Omega$. If $p=0$, then $f^{*}$ is holomorphic.

Proof. The case $p=0$ is a part of the cited result in [HR2]. Thus we may suppose that $p \neq 0$. Consider first the case in which $n=1$.

An easy computation shows that

$$
\int_{\mathbf{C}} \frac{|w|^{p-2}}{\left(1+|w|^{p}\right)^{2}} d m(w)=\frac{2 \pi}{|p|} .
$$

Take $z_{0} \in E$ arbitrarily. By assumption, there is an $r_{0}=r_{0}\left(p, f, z_{0}\right)>0$ such that

Since clearly

$$
\int_{B^{2}\left(z_{0}, r_{0}\right) \backslash E} \frac{|f(z)|^{p-2}}{\left(1+|f(z)|^{p}\right)^{2}}\left|f^{\prime}(z)\right|^{2} d m(z)<\frac{\pi}{|p|} .
$$

$$
\int_{f\left(B^{2}\left(z_{0}, r_{0}\right) \backslash E\right)} \frac{|w|^{p-2}}{\left(1+|w|^{p}\right)^{2}} d m(w) \leq \int_{B^{2}\left(z_{0}, r_{0}\right) \backslash E} \frac{|f(z)|^{p-2}}{\left(1+|f(z)|^{p}\right)^{2}}\left|f^{\prime}(z)\right|^{2} d m(z),
$$

$f \mid B^{2}\left(z_{0}, r_{0}\right) \backslash E$ omits a set of positive measure. Thus by a result of Kametani [No, Theorem 2, p. 10, and Remark, p. 11], $f$ has a meromorphic extension to $B^{2}\left(z_{0}, r_{0}\right)$.

The case in which $n \geq 2$ is proved using Fubini's theorem, Lemma 2.1(a) and Lemma 2.3. See [HR2, p. 301] and [Ri2, p. 549].

3.2. Corollary ([Pa, Théorème 20, p. 182] and [HR1, Theorem 3.4, p. 477]). Suppose that $\Omega$ is a domain in $C^{n}, n \geq 1$, and that $E \subset \Omega$ is closed in $\Omega$ and polar in $R^{2 n}$. Suppose that $f$ is holomorphic in $\Omega \backslash E$. If the (pluri)subharmonic function $\log ^{+}|f|$ has a harmonic majorant in $\Omega \backslash E$, then $f$ has a meromorphic extension to $\Omega$.

Proof. Because of the inequality $\log \left(1+x^{2}\right) \leq 2 \log ^{+}|x|+\log 2$, the (pluri)subharmonic function $\log \left(1+|f|^{2}\right)$ has a harmonic majorant in $\Omega \backslash E$. Proceeding then as in [Ri2, proof of Corollary 1, p. 549], one sees that the measure $\mu=$ $\Delta \log \left(1+|f|^{2}\right)$ has locally finite mass near $E$; i.e., for each domain $D$ relatively compact in $\Omega$, one has $\mu(D \backslash E)<\infty$. See also [Ce, p. 283]. On the other hand, computing the Laplacian, one easily gets

$$
\mu(D \backslash E)=4 \int_{D \backslash E} \frac{1}{\left(1+|f(z)|^{2}\right)^{2}} \sum_{j=1}^{n}\left|\frac{\partial f}{\partial z_{j}}(z)\right|^{2} d m(z)
$$

for each domain $D$ relatively compact in $\Omega$. Compare [HR2, p. 297], [Ri2, p. 549] and [Ya, p. 402]. Since $\mu(D \backslash E)<\infty$, the result follows then from Theorem 3.1. 
3.3. Remark. Note that the above proof for Corollary 3.2 applies for all $n \geq 1$. Our previous, more complicated proof [HR1, pp. 477-479] for the case $n \geq 2$ was based on Parreau's result (the case $n=1$ ) and on certain measure-theoretic results of Mattila and Sadullaev. For a third proof for the case $n=1$ see [Hej, Theorem 3, pp. 105-108].

3.4. Remark. A proof of Lehto's and Virtanen's result, Theorem 1.1 above, can be based on a special case of Theorem 3.1. As a matter of fact, this is essentially Järvi's method in his proof [Jä, Lemma 1, p. 1172]: he considers the special case when $n=1, \Omega=B^{2}(a, r) \subset \subset G, p=2$, and $E=\{a\} \cup N_{f}$. Here $N_{f}$ is the set of poles of $f$ in $G \backslash\{a\}$.

Next we give a generalization to [Jä, Theorem 2, p. 1174].

3.5. Theorem. Suppose that $\Omega$ is a domain in $C^{n}, n \geq 1$, that $E \subset \Omega$ is closed in $\Omega$, and that $H^{2 n-2}(E \cap K)<\infty$ for each compact set $K \subset \Omega$. Suppose that $f: \Omega \backslash E \rightarrow \mathbf{C}^{*}$ is a meromorphic function. If $f$ is normal, then $f$ has a meromorphic extension $f^{*}$ to $\Omega$.

Proof. We may suppose that $f \not \equiv \infty$. Write $E_{1}=E \cup N_{f}$, and note that $f$ is holomorphic in $\Omega \backslash E_{1}$. The result follows then at once with the aid of Lemma 2.1 (b), Lemma 2.2, Theorem 1.1, and Lemma 2.3.

3.6. If one wants the extension $f^{*}$ in Theorem 3.5 to be a meromorphic function from $\Omega$ to $\mathbf{C}^{*}$, then some additional restrictions must be imposed on the exceptional set $E$. This is seen from Järvi's example [Jä, Remark 2, p. 1174 ], which we recall here for the convenience of the reader.

Let $n \geq 3$. Set

$$
V_{j}=\left\{z=\left(z_{1}, \ldots, z_{n}\right) \in C^{n}: z_{j}=0\right\}
$$

and

$$
W_{1}=\left\{z=\left(z_{1}, \ldots, z_{n}\right) \in C^{n}: z_{1}-z_{2}=0\right\} .
$$

for each $j, 1 \leq j \leq n$. Then $V=V_{1} \cup V_{2} \cup W_{1}$ is an analytic subvariety in $C^{n}$ of codimension 1 . One sees at once that $f, f(z)=z_{1} / z_{2}$, is meromorphic in $C^{n}$ and is holomorphic and normal in $C^{n} \backslash V$. However, $f \mid C^{n} \backslash V$ does not extend to a meromorphic function from $C^{n}$ to $\mathbf{C}^{*}$.

In order to exclude such cases, Järvi considers the case in which the exceptional set $E=V$ is an analytic subvariety in $\Omega$ of codimension 1 whose singularities are normal crossings; that is, $\Omega \backslash V$ is locally biholomorphic to $\left[B^{2}(0,1) \backslash\{0\}\right]^{k} \times B^{2}(0,1)^{n-k}$ for some $k \in\{0,1, \ldots, n\}$. Below we propose one possibility for a less restrictive condition on the exceptional set. Since our method of proof is based on induction, our condition will be given on complex lines.

3.7. Suppose that $\Omega$ is an open set in $C^{n}, n \geq 1$. Let $\delta>0$. We say that a set $A \subset C^{n}$ is almost separately $\delta$-sparse in $\Omega$, or briefly $\delta$-sparse in $\Omega$, if the following conditions are satisfied. 
(i) If $n=1$, then

$$
|a-b| \geq \delta \cdot d(a, \partial \Omega)
$$

for all $a, b \in A \cap \Omega, a \neq b$.

(ii) If $n \geq 2$, then for each $k, 1 \leq k \leq n, A\left(Z_{k}\right)$ is $\delta$-sparse in $\Omega\left(Z_{k}\right)$ for $H^{2 n-2}$-almost all $Z_{k} \in C^{n-1}$.

A set $A$ is almost separately sparse in $\Omega$, or briefly sparse in $\Omega$, if $A$ is $\delta$-sparse in $\Omega$ for some $\delta>0$.

When $n=1$ and $\Omega=B^{2}(0,1)$ our condition is equivalent to the following well-known sparseness condition on the pseudohypert lic distance for the set $A$ : there is a $\delta^{\prime}>0$ such that

$$
\frac{|a-b|}{|1-a \bar{b}|} \geq \delta^{\prime}
$$

for all $a, b \in A \cap B^{2}(0,1), a \neq b$. See [CG2, p. 691, 694] and also [VH, p. 147], [Sa, p. 22].

It is easy to see that measurable sparse sets (in $\Omega$ ) are of Lebesgue measure zero. Sparse sets (in $\Omega$ ) can, of course, by very large. In fact, using a suitable sequence of Cartesian products of appropriate Cantor sets one easily gets a compact sparse set in $C^{n}$ whose Hausdorff dimension is $2 n$. There are also more surface-like examples: in $C^{2}$ there exists a compact set $K$ whose Hausdorff dimension is 4 such that for each $z=\left(z_{1}, z_{2}\right) \in C^{2}$ the sections $K\left(z_{1}\right)$ and $K\left(z_{2}\right)$ contain at most one point. The existence of such a set is based on [DF, Theorem 2, p. 118], see [HR2, p. 298]. For more natural examples, see 3.12 and 3.13 below.

3.8. Lemma. Suppose that $\Omega$ is a domain in $C^{n}, n \geq 2$, and that $A \subset \Omega$ is $\delta$-sparse in $\Omega$. Then for each $k, 1 \leq k \leq n, A\left(z_{k}\right)$ is $\delta$-sparse in $\Omega\left(z_{k}\right)$ for $H^{2}$-almost all $z_{k} \in \mathbf{C}$.

Proof. Since the assertion clearly holds when $n=2$, we may suppose that $n \geq 3$. Suppose that $1 \leq k \leq n$. Write, for each $j \neq k, 1 \leq j \leq n$,

$$
A_{j}=\left\{Z_{j} \in C^{n-1}: A\left(Z_{j}\right) \text { is not } \delta \text {-sparse in } \Omega\left(Z_{j}\right)\right\} \text {. }
$$

Since $H^{2 n-2}\left(A_{j}\right)=0$, we see with the aid of Fubini's theorem that $H^{2}\left(A_{j k}\right)=$ 0 , where

$$
A_{j k}=\left\{z_{k} \in \mathbf{C}: H^{2 n-4}\left(A_{j}\left(z_{k}\right)\right)>0\right\} .
$$

Write

$$
A_{k}^{*}=\bigcup_{j=1, j \neq k}^{n} A_{j k} .
$$

Then $H^{2}\left(A_{k}^{*}\right)=0$. It is easy to see that $A\left(z_{k}\right)$ is $\delta$-sparse in $\Omega\left(z_{k}\right)$ for all $z_{k} \in \mathbf{C} \backslash A_{k}^{*}$.

3.9. Theorem. Suppose that $\Omega$ is a domain in $C^{n}, n \geq 1$, and that $E \subset \Omega$ is closed in $\Omega$ and sparse in $\Omega$. Suppose that $f: \Omega \backslash E \rightarrow \mathbf{C}^{*}$ is a meromorphic 
function. If $f$ is normal, then $f$ extends to a meromorphic function $f^{*}: \Omega \rightarrow$ $\mathbf{C}^{*}$.

Proof. It is sufficient to show that $f$ has a spherically continuous extension to a neighborhood of each point $z_{0} \in E$. Because of Theorem 1.1, we may suppose that $n \geq 2$. We give an induction proof. One sees at once that the proof of Theorem 3.5 works also in the present case, i.e., when the exceptional set is sparse in $\Omega$. Thus $f$ has a meromorphic extension $f^{*}$ to $\Omega$. We may suppose that $f^{*} \not \equiv \infty$. Moreover, we may suppose that $E$ is $\delta$-sparse in $\Omega$, where $0<\delta<1$.

Take $z_{0} \in E$ and $R>0$ such that $\bar{D} \subset \Omega$, where $D=D\left(z_{0}, R\right)=$ $B^{2}\left(z_{1}^{0}, R\right) \times \cdots \times B^{2}\left(z_{n}^{0}, R\right)$. Let $C \geq 0$ be the order of normality of $f$. Choose $R_{1}>0$ and $M>1$ such that

$$
R_{1}<\frac{\delta R}{5} e^{-300 C^{2}}
$$

and

$$
\frac{4 \pi C^{2}}{M^{2}-1}<\frac{\pi}{150}
$$

Write $A=A_{1} \cap A_{2} \cap A_{3}$, where

$$
\begin{aligned}
& A_{1}=\left\{Z_{n} \in C^{n-1}: E\left(Z_{n}\right) \text { is } \delta \text {-sparse in } \Omega\left(Z_{n}\right)\right\}, \\
& A_{2}=\left\{Z_{n} \in C^{n-1}: N_{f^{*}}\left(Z_{n}\right) \text { is countable }\right\}, \\
& A_{3}=\left\{Z_{n} \in C^{n-1}: I_{f^{*}}\left(Z_{n}\right)=\varnothing\right\} .
\end{aligned}
$$

With the aid of Lemma 2.1 (b), one sees that $H^{2 n-2}\left(C^{n-1} \backslash A\right)=0$. Take $Z_{n} \in$ $A$ arbitrarily, and write $g=f_{Z_{n}}$. By Lemma $2.2, g$ is normal in $(\Omega \backslash E)\left(Z_{n}\right)$ and has therefore by Theorem 1.1 a meromorphic extension $g^{*}$ to $\Omega\left(Z_{n}\right)$. Clearly $g^{*}=f_{Z_{n}}^{*}$, where $f_{Z_{n}}^{*}: \Omega\left(Z_{n}\right) \rightarrow \mathbf{C}^{*}$,

$$
f_{Z_{n}}^{*}\left(z_{n}\right)=f^{*}\left(z_{n}, Z_{n}\right)=f^{*}\left(Z_{n}, z_{n}\right) .
$$

Consider first the case in which $E\left(Z_{n}\right) \cap B^{2}\left(z_{n}^{0}, R_{1}\right) \neq \varnothing$. Choose $z_{n}^{*} \in$ $E\left(Z_{n}\right) \cap B^{2}\left(z_{n}^{0}, R_{1}\right)$ arbitrarily; then $B^{2}\left(z_{n}^{*}, \delta\left(R-R_{1}\right)\right) \backslash\left\{z_{n}^{*}\right\} \subset(\Omega \backslash E)\left(Z_{n}\right)$. Because $g$ is normal also in $B^{2}\left(z_{n}^{*},(\delta / 2)\left(R-R_{1}\right)\right) \backslash\left\{z_{n}^{*}\right\}$, because the Kobayashi metric and the Poincare metric of $B^{2}\left(z_{n}^{*},(\delta / 2)\left(R-R_{1}\right)\right) \backslash\left\{z_{n}^{*}\right\}$ are the same, and because $C_{g \mid B^{2}\left(z_{n}^{*},(\delta / 2)\left(R-R_{1}\right)\right) \backslash\left\{z_{n}^{*}\right\}} \leq C_{g} \leq C$ by Lemma 2.2, one has

$$
\frac{\left|g^{* \prime}\left(z_{n}\right)\right|}{1+\left|g^{*}\left(z_{n}\right)\right|^{2}} \leq C \frac{1}{\left|z_{n}-z_{n}^{*}\right| \log \frac{\delta\left(R-R_{1}\right)}{2\left|z_{n}-z_{n}^{\prime}\right|}}
$$

for all $z_{n} \in B^{2}\left(z_{n}^{*},(\delta / 2)\left(R-R_{1}\right)\right) \backslash\left(\left\{z_{n}^{*}\right\} \cup N_{g^{*}}\right)$. See [LV, proof of Theorem 9, 
p. 63]. [Ah, p. 17] and [Jä, proof of Lemma 1, p. 1172]. Since $B^{2}\left(z_{n}^{*}, 2 R_{1}\right) \subset$ $B^{2}\left(z_{n}^{*},(\delta / 2)\left(R-R_{1}\right)\right)$, it follows from (3) that

$$
\int_{B^{2}\left(z_{n}^{*}, 2 R_{1}\right)} \frac{\left|g^{*^{\prime}}\left(z_{n}\right)\right|^{2}}{\left(1+\left|g^{*}\left(z_{n}\right)\right|^{2}\right)^{2}} d m\left(z_{n}\right) \leq \frac{2 \pi C^{2}}{\log \frac{\delta\left(R-R_{1}\right)}{4 R_{1}}} .
$$

Proceeding then as in the proof of Theorem 3.1, we see with the aid of (1) that

$$
m_{s p}\left(g^{*}\left(B^{2}\left(z_{n}^{0}, R_{1}\right)\right)\right) \leq m_{s p}\left(g^{*}\left(B^{2}\left(z_{n}^{*}, 2 R_{1}\right)\right)\right) \leq \frac{2 \pi C^{2}}{\log \frac{\delta\left(R-R_{\perp}\right)}{4 R_{1}}}<\frac{\pi}{150} .
$$

By $m_{s p}$ (the spherical measure in $\mathbf{C}^{*}$ ) we mean the following: If $H \subset \mathbf{C}^{*}$ is such that $H \backslash\{\infty\}$ is measurable in $\mathrm{C}$, then we write

$$
m_{s p}(H)=\int_{H \backslash\{\infty\}} \frac{1}{\left(1+|w|^{2}\right)^{2}} d m(w) .
$$

On the other hand,

$$
\begin{aligned}
& m_{s p}\left(B^{2}(0,1)\right)=\frac{\pi}{2}, \\
& m_{s p}\left(B^{2}(0,3) \backslash \bar{B}^{2}(0,2)\right)=\frac{\pi}{10}, \\
& m_{s p}\left(B^{2}(0,7) \backslash \bar{B}^{2}(0,6)\right)>\frac{\pi}{150} .
\end{aligned}
$$

Thus there is a number $\varepsilon>0$ independent of $Z_{n}$ such that $g^{*}$ omits in $B^{2}\left(z_{n}^{0}, R_{1}\right)$ three points $a_{g^{*}}, b_{g^{*}}, c_{g^{*}} \in \mathbf{C}$ such that

$$
q\left(a_{g^{*}}, b_{g^{*}}\right) q\left(b_{g^{*}}, c_{g^{*}}\right) q\left(c_{g^{*}}, a_{g^{*}}\right) \geq \varepsilon .
$$

(For example, $\varepsilon=1 / 125,000$ will do.)

Consider then the case in which $E\left(Z_{n}\right) \cap B^{2}\left(z_{n}^{0}, R_{1}\right)=\varnothing$. As above, $g$ is normal in $B^{2}\left(z_{n}^{0}, R_{1}\right)$ and $C_{g \mid B^{2}\left(z_{n}^{0}, R_{1}\right)} \leq C_{g} \leq C$. Thus,

$$
\frac{\left|g^{\prime}\left(z_{n}\right)\right|}{1+\left|g\left(z_{n}\right)\right|^{2}} \leq C \frac{2 R_{1}}{R_{1}^{2}-\left|z_{n}-z_{n}^{0}\right|^{2}}
$$

for all $z_{n} \in B^{2}\left(z_{n}^{0}, R_{1}\right) \backslash N_{g}$. (Recall that the Kobayashi metric and the Poincare metric of $B^{2}\left(z_{n}^{0}, R_{1}\right)$ are the same.) Using this inequality and (2), one gets

$$
m_{s p}\left(g\left(B^{2}\left(z_{n}^{0}, \frac{R_{1}}{M}\right)\right)\right) \leq \frac{4 \pi C^{2}}{M^{2}-1}<\frac{\pi}{150} .
$$

Proceeding again as above, one sees that $g$ omits in $B^{2}\left(z_{n}^{0}, R_{1} / M\right)$ three points $a_{g}, b_{g}, c_{g} \in \mathbf{C}$ such that

$$
q\left(a_{g}, b_{g}\right) q\left(b_{g}, c_{g}\right) q\left(c_{g}, a_{g}\right) \geq \varepsilon,
$$

where $\varepsilon>0$ is the same number as above. Because of (4) and (5), we see by Lemma 2.4 that the family

$$
\left\{f_{Z_{n}}^{*}: B^{2}\left(z_{n}^{0}, \frac{R_{1}}{M}\right) \rightarrow \mathrm{C}^{*}: Z_{n} \in A \cap D\left(z_{n}^{0}\right)\right\}
$$


of meromorphic functions is spherically equicontinuous. (As for the notation, recall that $D\left(z_{n}^{0}\right)$ is the section of $D$.)

To conclude the proof we show that $f$ has a spherically continuous extension to $D_{1}=B^{2}\left(z_{1}^{0}, R\right) \times \cdots \times B^{2}\left(z_{n-1}^{0}, R\right) \times B^{2}\left(z_{n}^{0}, R_{1} / M\right)$. To prove this, it is clearly sufficient to show that the following condition is fulfilled: for each $Z_{n}^{\prime} \in D_{1}\left(z_{n}^{0}\right)$ and for each sequence $Z_{n}^{j} \rightarrow Z_{n}^{\prime}, Z_{n}^{j} \in A \cap D_{1}\left(z_{n}^{0}\right), j=1,2, \ldots$, the sequence

$$
f_{Z_{n}^{j}}^{*}: B^{2}\left(z_{n}^{0}, \frac{R_{1}}{M}\right) \rightarrow \mathbf{C}^{*}, j=1,2, \ldots,
$$

of meromorphic functions converges spherically $c$-uniformly. (See, e.g., [Ri1, Lemma 2.6, p. 48]; in this elementary lemma one can of course equally well use a spherical metric instead of a Euclidean metric.) By [Vä, Theorem 20.3, p. 68], it is thus sufficient to show that the sequence (6) converges in a dense subset of $B^{2}\left(z_{n}^{0}, R_{1} / M\right)$.

We consider first the case when $n=2$. Since $H^{2 n-4}\left(I_{f} * \cap K\right)<\infty$ for each compact set $K \subset \Omega, I_{f^{*}}\left(z_{n}\right)=\varnothing$ for $H^{2}$-almost all $z_{n} \in B^{2}\left(z_{n}^{0}, R_{1} / M\right)$. Thus the desired convergence follows.

Suppose then that $n \geq 3$. With the aid of Lemma 3.8 and Lemma 2.1 (b), we find a set $B \subset B^{2}\left(z_{n}^{0}, R_{1} / M\right)$ such that $H^{2}\left(B^{2}\left(z_{n}^{0}, R_{1} / M\right) \backslash B\right)=0$ and such that for each $z_{n} \in B$ the set $E\left(z_{n}\right)$ is $\delta$-sparse in $\Omega\left(z_{n}\right)$ and $H^{2 n-4}\left(N_{f} \cdot\left(z_{n}\right) \cap K\right)<\infty$ for each compact set $K \subset \Omega\left(z_{n}\right)$. Choose $z_{n} \in B$ arbitrarily. Then $h=f_{z_{n}}:(\Omega \backslash E)\left(z_{n}\right) \rightarrow \mathbf{C}^{*}$ is clearly a meromorphic function. Since $h$ is normal by Lemma 2.2 , we see by the induction hypothesis that $h$ extends to a meromorphic function $h^{*}: \Omega\left(z_{n}\right) \rightarrow \mathbf{C}^{*}$. Clearly $h^{*}\left(Z_{n}\right)=f^{*}\left(z_{n}, Z_{n}\right)=f^{*}\left(Z_{n}, z_{n}\right)=f_{Z_{n}}^{*}\left(z_{n}\right)$ for each $Z_{n} \in \Omega\left(z_{n}\right) \backslash I_{f^{*}}\left(z_{n}\right)$. Recalling then that $Z_{n}^{j} \in A, j=1,2, \ldots$, and the definition of $A$, we see that $Z_{n}^{j} \notin I_{f^{*}}\left(z_{n}\right)$. Thus $f_{Z_{n}^{j}}^{*}\left(z_{n}\right) \rightarrow h^{*}\left(Z_{n}^{\prime}\right)$ as $j \rightarrow \infty$. Hence we have shown that the sequence (6) converges for each $z_{n} \in B$. Therefore the induction step is done and the proof is complete.

3.10. Corollary. Suppose that $\Omega$ is a domain in $C^{n}, n \geq 1$, that $E$ is closed in $\Omega$, and that for each $k, 1 \leq k \leq n$, card $E\left(Z_{k}\right) \leq 1$ for $H^{2 n-2}$-almost all $Z_{k} \in C^{n-1}$. Suppose that $f: \Omega \backslash E \rightarrow \mathbf{C}^{*}$ is a meromorphic function. If $f$ is normal, then $f$ extends to a meromorphic function $f^{*}: \Omega \rightarrow \mathbf{C}^{*}$.

3.11. In concluding we give two examples. These give natural exceptional sets in the considered setting of meromorphic functions with values in $C^{*}$, which satisfy our condition in Corollary 3.10 , but which are such that the extension result [Jä, Theorem 1, p. 1173] cannot be applied.

3.12. Example. Set $n=3 ; V=V_{1} \cup V_{2} \cup V_{3} \cup W_{2}$, where $V_{j}, j=1,2,3$, are as in 3.6 above and

$$
W_{2}=\left\{z=\left(z_{1}, z_{2}, z_{3}\right) \in C^{3}: z_{1}=z_{2}=z_{3}\right\} \text {. }
$$


Then $V$ is an analytic subvariety in $C^{3}$. However, $V$ is not contained in any analytic subvariety $V^{\prime}$ in $C^{3}$ of codimension 1 whose singularities are normal crossings. On the other hand, $V$ is sparse in $C^{3}$.

Note further that $V$ is a natural exceptional set for meromorphic functions in our setting: for example, the meromorphic function $f: C^{3} \backslash V \rightarrow \mathbf{C}^{*}$,

$$
f\left(z_{1}, z_{2}, z_{3}\right)=e^{-1 / z_{1}}+e^{-1 / z_{2}}+e^{-1 / z_{3}}+\frac{z_{1}-z_{3}}{z_{2}-z_{3}}
$$

cannot be extended to any larger set as a meromorphic function with values in $\mathrm{C}^{*}$.

3.13. Example. Set $n=2$ and $\Omega=B^{2}(0,1) \times B^{2}(0,1)$. Choose a sequence $b_{k}=\left(b_{1}^{k}, b_{2}^{k}\right) \in \Omega, k=1,2, \ldots$, in such a way that $b_{k} \rightarrow(0,0)$ as $k \rightarrow \infty$ and that the set

$$
E=\left\{b_{k}=\left(b_{1}^{k}, b_{2}^{k}\right) \in \Omega: k=1,2, \ldots\right\} \cup\left[B^{2}(0,1) \times\{0\}\right] \cup\left[\{0\} \times B^{2}(0,1)\right]
$$

is not contained in any analytic subvariety in $\Omega$. As a matter of fact, using an antithesis and, for example, [Her, Corollary 3, p. 36; Remark, p. 33; Remark, p. 44; Theorem 6, p. 33; and part of the proof of Theorem 1 (ii), p. 11] one sees that one possibility for this is the following: choose a sequence of distinct points $a_{j} \in B^{2}(0,1), j=1,2, \ldots$, such that $a_{j} \rightarrow 0$ as $j \rightarrow \infty$ and write

$$
\begin{aligned}
& b_{1}^{k}=a_{j}, \text { when } \frac{(j-1) j}{2}<k \leq \frac{j(j+1)}{2} \text { for some } j \in \mathbf{N}, \\
& b_{2}^{k}=a_{j+k-\frac{(j-1) j}{2}}, \text { when } \frac{(j-1) j}{2}<k \leq \frac{j(j+1)}{2} \text { for some } j \in \mathbf{N},
\end{aligned}
$$

for $k=1,2, \ldots$. Nevertheless, $E$ is clearly sparse in $\Omega$.

Again, our set $E$ is a natural exceptional set in the situation considered. In fact, suppose that

$$
\sum_{j=1}^{\infty}\left|c_{j}\right|<\infty
$$

Then it is routine to check that $f$,

$$
f\left(z_{1}, z_{2}\right)=e^{-1 / z_{1}}+e^{-1 / z_{2}}+\sum_{j=1}^{\infty} c_{j} \frac{z_{1}-b_{1}^{j}}{z_{2}-b_{2}^{j}}
$$

is a meromorphic function from $\Omega \backslash E$ to $\mathrm{C}^{*}$, which, however, cannot be extended to any larger set as a meromorphic function with values in $\mathbf{C}^{*}$.

\section{REFERENCES}

[Ah] L. V. Ahlfors, Conformal invariants. Topics in geometric function theory, McGraw-Hill, New York, 1973.

[Ca] C. Carathéodory, Theory of functions, vol. 2, Chelsea, New York, 1960.

[Ce] U. Cegrell, Removable singularity sets for analytic functions having modulus with bounded Laplace mass, Proc. Amer. Math. Soc. 88 (1983), 283-286. 
[CG1] J. A. Cima and I. R. Graham, On the extension of holomorphic functions with growth conditions across analytic subvarieties, Michigan Math. J. 28 (1981), 241-256.

[CG2] _ Removable singularities for Bloch and BMO functions, Illinois J. Math. 27 (1983), 691-703.

[CK] J. A. Cima and S. G. Krantz, The Lindelöf principle and normal functions of several complex variables, Duke Math. J. 50 (1983), 303-328.

[DF] R. O. Davies and H. Fast, Lebesgue density influences Hausdorff measure; Large sets surfacelike from many directions, Mathematika 25 (1978), 116-119.

[Fe] H. Federer, Geometric measure theory, Springer-Verlag, Berlin, 1969.

[Hej] D. A. Hejhal, Linear extremal problems for analytic functions, Acta Math. 128 (1972), 91-122.

[Her] M. Hervé, Several complex variables, 2nd ed., Oxford University Press, New Delhi, 1987.

[HR1] J. Hyvönen and J. Riihentaus, On the extension in the Hardy classes and in the Nevanlinna class, Bull. Soc. Math. France 112 (1984), 469-480.

[HR2] __ Removable singularities for holomorphic functions with locally finite Riesz mass, J. London Math. Soc. 35 (1987), 296-302.

[Jä] P. Järvi, An extension theorem for normal functions, Proc. Amer. Math. Soc. 103 (1988), 1171-1174.

[Ki] P. Kiernan, Extensions of holomorphic maps, Trans. Amer. Math. Soc. 172 (1972), 347-355.

[LV] O. Lehto and K. I. Virtanen, Boundary behaviour and normal meromorphic functions, Acta Math. 97 (1957), 47-65.

[No] K. Noshiro, Cluster sets, Springer-Verlag, Berlin, 1960.

[Pa] M. Parreau, Sur les moyennes des fonctions harmoniques et analytiques et la classification des surfaces de Riemann, Ann. Inst. Fourier (Grenoble) 3 (1951), 103-197.

[Ri1] J. Riihentaus, Removable singularities of analytic functions of several complex variables, Math. Z. 158 (1978), 45-54.

[Ri2] _ Removable singularities in the Nevanlinna class and in the Hardy classes, Proc. Amer. Math. Soc. 102 (1988), 546-550.

[Sa] D. Sarason, Function theory on the unit circle, Lecture Notes, Virginia Polytech. Inst. and State Univ., Blacksburg, VA, 1978.

[Sh] B. Shiffman, On the removal of singularities of analytic sets, Michigan Math. J. 15 (1968), 111-120.

[Vä] J. Väisälä, Lectures on n-dimensional quasiconformal mappings, Springer-Verlag, Berlin, 1971.

[VH] S. A. Vinogradov and V. P. Havin, Free interpolation in $\mathrm{H}^{\infty}$ and in other certain classes of functions, I, J. Soviet Math. 9 (1978), 137-171.

[Ya] S. Yamashita, Functions of uniformly bounded characteristic on Riemann surfaces, Trans. Amer. Math. Soc. 288 (1985), 395-412.

Department of Mathematics, University of Oulu, SF-90570 Oulu, Finland 